\title{
PAUL TANNERY ET JOSEPH NEEDHAM DEUX PLAIDOYERS POUR UNE HISTOIRE GÉNÉRALE DES SCIENCES
}

\author{
Karine Chemla et Jeanne PeIFFER
}

\begin{abstract}
Résumé: Alors qu'ils présentent tous deux, sous l'impulsion de la tentative comtienne, des histoires globales des sciences, Paul Tannery et Joseph Needham mettent en place des cadres historiographiques de fait distincts. Tannery fonde son histoire générale sur la notion de civilisation, dont la science est un élément intégrant. L'outil méthodologique qu'il utilise pour dégager les traits saillants qui caractérisent une civilisation donnée à une époque donnée sous l'aspect de la science est la synthèse. Needham part de la science moderne, et se donne pour objectif de mettre en évidence qu'elle fut constituée par un processus de synthèse des apports de différentes traditions. Pour lui, la formation de ce savoir moderne a procédé d'un effort continu qui a tour à tour été mené par les divers groupes composant l'humanité. Tannery, en revanche, est très préoccupé par les ruptures et les crises, qui peuvent être à l'origine de la disparition de pans entiers du savoir, mais aussi d'intenses périodes de création. Ce qu'il est convenu d'appeler « révolution scientifique » prend alors un sens très différent pour les deux historiens.
\end{abstract}

Mots-CLÉs : synthèse, historiographie, révolution scientifique, Chine.

ABSTRACT : Whereas, under the influence of Comte's endeavour, they both present global histories of science, in fact, Paul Tannery and Joseph Needham use distinct historiographical frameworks. Tannery grounds his general history on the notion of civilisation, of which science is a component. The methodological tool he uses to highlight the salient features that characterise a given civilisation at a given time as regards science is synthesis. Needham takes modern science as his starting point, and aims at bringing to light that it was constituted by a synthesis of the contributions of different traditions. For him, the shaping of this modern knowledge proceeded from a continuous effort that, in turn, was carried out by the various groups composing mankind. In contrast, Tannery is preoccupied by ruptures and crises, which can cause the disappearance of entire domains of knowledge, but also intense periods of creation. What is conventionally called the "scientific revolution " thus takes a very different meaning for the two historians.

KEYWORDS : synthesis, historiography, scientific revolution, China.

Revue de synthèse : $4^{\mathrm{e}}$ sér., ${ }^{\text {os }}$ 2-3-4, avr.-déc. 2001, p. 367-392. 
ZuSAMmENFASSUnG : Beide geben zwar, wohl unter dem Einfluss von Comte, allgemeine Darstellungen von Wissenschaftsgeschichte, aber der von Paul Tannery und Joseph Needham gespannte historiographische Rahmen ist in beiden Fällen grundverschieden. Tannery fundiert seine allgemeine Wissenschaftsgeschichte auf den Begriff der Zivilisation, wovon die Wissenschaft ein Bestandteil ist. Das methodologische Instrument, das er benutzt um die charakteristischen Züge einer gegebenen Zivilisation zu einem gegebenen Zeitpunkt in Bezug auf Wissenschaft herauszuarbeiten, ist die Synthese. Needhams Ausgangspunkt ist die moderne Wissenschaft und sein Ziel ist es zu beweisen, dass sie sich durch einen synthetischen die Beiträge verschiedener Traditionen integrierenden Prozess herausgebildet hat. Modernes Wissen ist demnach entstanden aus einem stetigen Aufwand, der abwechselnd von verschiedenen Menschengruppen geleistet wurde. Tannery hingegen beschäftigen Brüche und Krisen, welche das Verschwinden ganzer Wissenszweige verursachen, aber auch am Anfang intensiver kreativer Perioden stehen können. Was üblicherweise mit wissenschaftlicher Revolution bezeichnet wird, bekommt dadurch bei beiden Historikern einen unterschiedlichen Inhalt.

STICHWÖRTER: Synthese, Geschichtsschreibung, wissenschaftliche Revolution, China.

Karine ChEMLA, née en 1957, chercheur au Centre national de la recherche scientifique, équipe REHSEIS (CNRS et Université Paris 7), travaille sur l'histoire des mathématiques, tout particulièrement en Chine ancienne. Elle est responsable, avec François Martin (École pratique des hautes études), de la revue d'études extrême-orientales, Extrême-Orient, Extrême-Occident, et est rédactrice en chef adjointe de la Revue d'histoire des mathématiques.

Adresse : REHSEIS, CNRS et Université Paris 7, UFR GHSS, Centre Javelot, Université Paris 7, 2 pl. Jussieu, F-75005 Paris.

Courrier électronique : chemla@paris7.jussieu.fr

Jeanne PeifFer, née en 1948, est historienne des mathématiques au centre Alexandre-Koyré (École des hautes études en sciences sociales-Centre national de la recherche scientifiqueMuséum national d'histoire naturelle). Elle collabore avec Karine Chemla à la Revue d'histoire des mathématiques qu'elle dirige.

Adresse : Centre Alexandre-Koyré (CNRS), 27 rue Damesme, F-75013 Paris.

Courrier électronique : peiffer@damesme.cnrs.fr 
Les deux noms de Paul Tannery et Joseph Needham sont associés à des projets pour le moins différents en histoire des sciences. Si nous avons pourtant choisi de les confronter, en l'honneur d'Ernest Coumet, c'est pour faire revivre, le temps d'un article, l'atmosphère d'un séminaire, ouvert au débat, où nombre de positions historiographiques furent examinées et disséquées. Tannery et Needham y furent donc, parmi de nombreux autres, régulièrement convoqués, et nous voudrions ici mettre en discussion, sous un angle bien précis, les programmes qu'ils ont respectivement assignés à une histoire générale des sciences : pour différents qu'ils soient, en effet, leurs projets historiographiques, d'une envergure qui peut surprendre aujourd'hui, accordent tous deux une place centrale à une forme de synthèse. Nous nous donnons ici pour objectif de spécifier par une présentation contrastée ce que chacun entendait par ce terme et d'éclairer comment l'un et l'autre, ce faisant, s'inscrivaient à leur manière dans une époque.

\section{DEUX FORMATIONS SCIENTIFIQUES}

Une des «figures patronymiques » de la communauté des historiens des sciences, comme l'a justement souligné Ernest Coumet ${ }^{1}$, Paul Tannery (1843-1904) est un peu paradoxalement un amateur en matière d'histoire des sciences, mais un amateur « de génie ${ }^{2} »$ selon Ulrich von Wilamowitz. Polytechnicien, ingénieur des tabacs, Tannery fit en effet une carrière de commis de l'État (dans les manufactures de tabac de Lille, Bergerac, Bordeaux, Le Havre, Tonneins et Pantin). Helléniste autodidacte, il s'est très tôt intéressé à la philologie classique et discuta philosophie et mathématiques avec son frère Jules et avec Émile Boutroux. Son premier séjour bordelais (1874-1877), durant lequel il a pu rencontrer Louis Liard et Jules Hoüel, dont le rôle de passeur en matière d'histoire des mathématiques n'a pas été suffisamment apprécié, eut une influence durable sur l'orientation des travaux de Tannery. C'est à Bordeaux qu'il publia, dès 1876, ses pre-

1. Coumet, 1981, p. 87. Ernest Coumet donne quelques exemples d'un riche florilège de citations célébrant l'action pionnière de Tannery en faveur de l'histoire des sciences. Qu'il nous soit permis d'en ajouter quelques-uns. Ainsi, Federigo Enriques, préface à TANNERY, 1930 , p. XVIII, ne voit « qu'un petit nombre de pionniers qui aient préparé les voies actuelles ». SARTON, 1954, p. 321, en dit : «Quand on écrira l'histoire de nos études il faudra y donner une toute première place à Paul Tannery. »

2. Cité d'après Coumet, 1981, p. 87. 
miers travaux historiques. Dès cette époque, il consacra tous ses loisirs à la recherche en histoire des sciences exactes, ses champs de prédilection étant les mathématiques grecques et byzantines d'une part, les mathématiques dites du Grand Siècle de l'autre. Il est inutile de rappeler ici les deux grands monuments de l'édition qu'il nous a laissés sous la forme des œuvres complètes de René Descartes et de Pierre Fermat.

Ses travaux unanimement applaudis lui ont apporté une immense reconnaissance intellectuelle et l'ont inséré dans un réseau européen largement étendu. Avec Moritz Cantor, Gustav Eneström et Gino Loria notamment, Tannery s'efforça de développer l'histoire des mathématiques en tant que discipline autonome et d'organiser celle des sciences à l'échelle internationale (en créant des sections autonomes dans les congrès, des revues, des enseignements et des commissions de coordination). Il était un familier des Danois Johan Ludvig Heiberg et Hieronymus G. Zeuthen, qui lui ont rendu hommage à titre posthume en assumant la responsabilité scientifique de ses Mémoires scientifiques, patiemment réunis par son épouse Marie Tannery-Prisset.

C'est à titre de seconde carrière, mais selon une autre modalité, que Joseph Needham (1900-1995) choisit de se consacrer à l'histoire des sciences en Chine. Biologiste de formation, féru d'histoire et de philosophie des sciences, pétri de lectures théologiques, il appartient à ce groupe de biologistes marxistes de Cambridge que devait marquer de manière indélébile la forte délégation soviétique au second congrès international d'Histoire des sciences à Londres en $1931^{3}$. Pourtant, ce n'est pas à cette rencontre, mais à l'arrivée de trois étudiants chinois à Cambridge en 1937 que Needham fait lui-même remonter le basculement de son existence, alors qu'il a déjà derrière lui une brillante carrière de chimiste de l'embryologie. Il se prend de passion pour la Chine, se met au chinois et se voit de ce fait tout naturellement choisi pour être envoyé en 1942, et pour quatre ans, à Chongqing (Chine) où la Royal Society le charge d'assurer le contact avec les scientifiques chinois repliés du fait de l'invasion japonaise. Il met ce temps à profit pour amasser des documents qui fourniront les premiers matériaux de ce qui devait devenir Science and civilisation in China. Après un détour par la politique scientifique, à l'Unesco, de 1946 à 1948, il se réinstalle définitivement à Cambridge, désormais dans la peau d'un historien des sciences en Chine pour le reste de sa vie, et y attire les collaborateurs du monde entier dans un institut entièrement organisé, dans ses res-

3. WeRSKEY, 1988, donne une biographie collective de ce groupe qui comportait, outre Joseph Needham, John D. Bernal, John B. S. Haldane, Lancelot Hogben, et Hyman Levy. Il y détaille tout particulièrement l'impact de cette délégation. Voir, également, HorolENSHAw, 1973, l'autobiographie publiée par Needham sous pseudonyme. 
sources tant humaines que matérielles, autour du grand œuvre. La publication, continue depuis 1954, des nombreux volumes de Science and civilisation in China eut un immense impact. Elle révéla à nombre de lecteurs occidentaux l'existence de traditions scientifiques et techniques en Chine, mais elle contribua également à développer leur étude dans l'empire du Milieu même.

\author{
RECONSTRUCTION ET SYNTHÈSE \\ LE PROJET HISTORIOGRAPHIQUE DE PAUL TANNERY
}

Lorsqu'en 1900, dans le tout premier tome de la Revue de synthèse (historique, pour être fidèle au titre d'alors) et sous l'impulsion de son fondateur Henri Berr, Tannery réfléchit au développement possible de l'histoire des mathématiques après la publication de la monumentale histoire de Cantor, il lui juxtapose le «remarquable effort de synthèse historique ${ }^{4}$ » accompli par Auguste Comte dans ses Leçons de philosophie positive. Cet effort a abouti, selon Tannery, à des résultats « qui peuvent servir de point de départ assuré pour tout travail dans le même sens ${ }^{5}$ ». Et c'est dans cette philosophie, qu'il s'est assimilée à 22 ans, qu'il a lui-même puisé les principales motivations pour ses travaux « dont le but était de vérifier et de préciser ses idées [de Comte] sur l'Histoire des Sciences ${ }^{6}{ } »$. S'il a été amené à formuler des réserves et à amender la fameuse loi des trois états — théologique, métaphysique et positif — par lesquels auraient nécessairement passé toutes les sciences, il a surtout retenu de l'approche de Comte la visée générale, synthétique et même vulgarisatrice ${ }^{7}$, ainsi que la nécessité de développer l'histoire des sciences et de lui donner une place dans l' « histoire de la civilisation », eu égard surtout à l'importance grandissante que les sciences prennent dans la vie des sociétés.

Mais avant de rassembler, au tout début du $\mathrm{xx}^{\mathrm{e}}$ siècle, ses réflexions sur la synthèse, Tannery s'est appliqué, dans de très nombreuses études spécialisées, à en réunir des matériaux. Mettant les origines de la cosmologie, de l'astronomie et de la géométrie au centre de ses premiers intérêts d'historien des sciences, il a donné des travaux analytiques, des éditions et des tra-

4. TANNERY, 1912-1950, t. X, p. 22.

5. TANNERY, 1912-1950, t. X, p. 22.

6. TANNERY, 1912-1950, t. X, p. 134. Affirmée par Tannery à maintes reprises, l'influence des idées d'Auguste Comte sur lui mériterait à elle seule un travail circonstancié.

7. On lira avec intérêt Grange, 2000, chap. II. Elle y discute le lieu d'où considérer les sciences après l'effondrement du point de vue transcendant ou théologico-moral sur elles, et surtout la solution que Comte a proposée dans son système positiviste. 
ductions alliant recherche minutieuse et érudition solide. Comme l'a très justement formulé Federigo Enriques, «c'est par l'effort de reconstruction et de synthèse que l'œuvre de Tannery manifeste son originalité propre ${ }^{8} »$. Par reconstruction, il faut ici entendre une véritable méthode que Tannery applique aux textes scientifiques. Il convient d'abord de réunir une documentation la plus riche possible, qui sera mise à la disposition du lecteur en la traduisant le cas échéant. Puis de soumettre les sources à la critique en fonction de leur caractère et surtout de leur période. Tannery se méfie en effet des témoignages transmis par des époques qu'il qualifie de décadence scientifique et préfère, pour l'interprétation des textes étudiés, faire le pari de leur cohérence, reconstituée en utilisant ses propres connaissances scientifiques. Lorsque les informations positives font défaut pour restituer un procédé ou un raisonnement perdus, il y supplée par des reconstructions conjecturales plausibles, qu'il est par ailleurs toujours prêt à remettre en cause, à discuter, à affiner ou à écarter pour les remplacer par d'autres ${ }^{9}$.

Cette méthode est mise en œuvre dans Pour l'histoire de la science hellène. De Thalès à Empédocle (paru en 1887), dont le but visé est de « donner une sorte de complément à l'histoire des origines de la philosophie ${ }^{10}{ }$. Les premiers penseurs grecs étant considérés comme des philosophes, ils ont surtout été étudiés par ces derniers. Tannery, en mettant au jour les éléments de science - trace des observations, des expérimentations et des notions scientifiques - qui se trouvent dans ces doctrines, a redonné à ces penseurs leur qualité de savants et leur place dans l'histoire de la pensée scientifique. Examinées à la lumière d'une approche méthodologique radicalement différente et d'un point de vue inédit, ces doctrines, qui ne nous sont parvenues que par des témoignages tardifs, livrent un autre type d'histoire. Tannery en est pleinement conscient.

Un de ses résultats peut être formulé comme suit : la science grecque n'était aucunement l'aboutissement d'une lente évolution, d'un progrès continu de la pensée. Selon Tannery, elle a surgi brusquement, sans préparation, au v ${ }^{\mathrm{e}}$ siècle av. J.-C. Lorsque la période hellène s'ouvre, nous dit-il, « il n'y a encore ni science ni même aucune idée de ce que peut ou doit être la science; lorsqu'elle se ferme, deux immortels monuments sont debout $^{11} \gg$ (Hippocrate, Aristote). Fille de l'hellénisme, la science antique

8. Préf. à TANNERY, 1930, p. XIX.

9. Voici comment ITARD, 1954, p. 332, a décrit la méthode de Tannery : «Il trouve des documents inédits, il les édite, les traduit, les éclaire par des notes brèves et précises. Lorsque les passages obscurs sont rebelles à cette méthode, il met en œuvre toutes ses connaissances mathématiques pour suppléer par des conjectures plausibles à l'absence de précisions authentiques. Mais il sait toujours qu'une reconstitution conjecturale n'est jamais définitive, et laisse la question ouverte aux recherches ultérieures. »

10. TANNERY, 1887, p. 13.

11. TANNERY, 1887 , p. 1 . 
en partagera le sort, c'est-à-dire qu'elle disparaîtra avec lui. Comme Pierre Boutroux ${ }^{12}$ l'a remarqué, de nombreux écrits de Tannery tournent autour de cette problématique de la décadence et de ses causes, des crises qui font périr la science, mais peuvent aussi avoir pour effet de créer des disciplines et des méthodes nouvelles.

Pour Tannery, seule la science constituée en spécialité peut être objet d'histoire des sciences ${ }^{13}$. Le critère pour une telle spécialisation semble être un critère de professionnalisation, dans le sens que des individus, même isolés, peuvent ou doivent se consacrer exclusivement à cette science s'ils veulent obtenir des résultats et la faire avancer.

Ainsi, le calcul ou la géométrie pratique, par exemple, qui se développent sous la pression des besoins techniques ou artistiques, sont écartés de l'histoire des mathématiques, alors que leur développement est éminemment susceptible d'intéresser une histoire plus générale de la civilisation. Mais, selon Tannery, ils ne contribuent en rien au progrès des sciences (constituées), ainsi qu'en témoigne la citation : « [...] la science ne se développe que lorsqu'elle est cultivée pour elle-même ${ }^{14} \gg$.

Cette limitation de la notion de science amène Tannery à distinguer, pour chaque science, une période préscientifique, c'est-à-dire une période «dans laquelle les connaissances se développent au fur et à mesure des besoins de la technique, avant d'être reliées par une théorie et munies successivement de preuves complètes et décisives ${ }^{15} \gg$. Comment situer cette période par rapport aux trois états instaurés par la loi sur l'ordre des progrès d'Auguste Comte? Tannery ne se prononce pas, bien qu'il l'introduise pour illustrer les difficultés dans l'applicabilité de la loi comtienne. La notion de période préscientifique convient mieux (que l'état théologique) à la description de ce qu'il a pu observer de l'évolution des mathématiques pures. Ainsi, les superstitions attachées aux nombres n'apportent rien à l'histoire de l'arithmétique et peuvent en être écartées.

Cette conception restrictive de la science objet d'histoire motive sans doute le choix que fait Tannery de ses thèmes de recherche. Il s'intéresse avant tout à des périodes de renouveau théorique, les origines de la science grecque et la renaissance des mathématiques au XVII ${ }^{\mathrm{e}}$ siècle, entre lesquels il situe «quatorze siècles d'asservissement scientifique ${ }^{16}{ }$. C'est la distinction entre géométrie théorique et géométrie pratique qui lui permet d'écarter ces quatorze siècles. La première s'étant éclipsée, seule la

12. Boutroux, 1938, p. 698.

13. En fait, il faudrait dire d'histoire particulière ou spéciale des sciences. Pour la distinction entre histoires particulières et histoire générale des sciences, voir infra, p. 374-377. Cf. aussi Coumet, 1981, et PeIfFer, à paraître.

14. TANnery, 1893, cité par Sarton, 1938, p. 664.

15. TANnERy, 1912-1950, t. X, p. 25.

16. Cité par Boutroux, 1938, p. 664. 
seconde a pu progresser, mais dans l'incapacité de poser de nouveaux problèmes à la science, elle n'a eu aucune influence sur le développement de la science théorique ${ }^{17}$.

Pour les mathématiques, les grands cadres historiographiques sont ainsi fixés et fondés sur la rupture entre mathématiques pratiques et mathématiques abstraites, les premières (arpentage, art du dessin, constructions) étant présentes chez tous les peuples et en rapport avec leur degré de civilisation, les secondes étant incontestablement une création du « génie grec ». Elles ont connu une période de décadence (en Occident) pour finalement disparaître et avec elles l'idée même de science. Du côté de l'Orient, les Arabes, auxquels est systématiquement déniée toute originalité, sont importants pour l'histoire des mathématiques «parce qu'ils ont transmis à l'Occident latin, avec les éléments de la science grecque, les procédés de calcul par les chiffres modernes, y compris ceux de l'algèbre ${ }^{18} »$. Avec la renaissance des mathématiques $\mathrm{au} \mathrm{XVII}^{\mathrm{e}}$ siècle, "c'est bien la science moderne qui se dégage avec ses traits définitifs ${ }^{19}{ }$.

Eu égard à l'importance que Tannery accorde à la science constituée, on ne sera pas étonné de le voir œuvrer très activement, par ses écrits et ses interventions orales dans les congrès internationaux ${ }^{20}$, pour la constitution de l'histoire des sciences en discipline autonome (complémentaire par rapport à la philosophie) et digne d'être enseignée à tous les niveaux de l'instruction. Tannery définit, en effet, pour chaque formation et chaque degré son «type » d'histoire. À côté des histoires particulières faites par les spécialistes des sciences dont on fait l'histoire et enseignées dans le second degré par les professeurs correspondants, il milite pour la composition d'une histoire à caractère synthétique : l'histoire générale des sciences.

C'est d'ailleurs dans un contexte lié à l'enseignement universitaire que Tannery a mis au clair sa conception d'une telle histoire et de ses liens avec les histoires particulières (disciplinaires par exemple). Candidat à la chaire d'histoire générale des sciences ${ }^{21}$ créée en 1892 au Collège de France et vacante en 1903, Tannery, classé en tête de liste par ses pairs, a rédigé sa leçon inaugurale. Comme la chaire lui a finalement été refusée par le

17. Dans les notices rédigées pour l'encyclopédie éditée par Ernest LAVISSE et Alfred RAMBAUD, Histoire générale du IV siècle à nos jours, Paris, 1893-1901 (voir infra n. 30), TANNERY, 1912-1950, t. XVII, p. 125, après avoir développé cet argument, conclut brutalement : «La science proprement dite n'existe donc pas encore au Moyen Âge. »

18. TANNERY, 1912-1950, t. VI, p. 576.

19. TANNERY, 1912-1950, t. XVII, p. 167.

20. Pour une étude de ces actions, notamment dans les congrès internationaux de 1900 à 1904, voir Coumet, 1981 et aussi la contribution de Enrico Castelli GatTinara à ce volume, supra, p. 347-365.

21. Sur l'histoire de cette chaire et l'échec de la candidature de Tannery, voir en particulier: Sarton, 1947, Paul, 1976, Coumet, 1981, et Petit, 1995. 
ministre, la leçon ne fut pas tenue, mais elle fut néanmoins publiée ${ }^{22}$, à titre posthume, par Henri Berr dans la Revue de synthèse historique de l'année 1904.

Pour Tannery, comme pour Comte, l'histoire des sciences est partie intégrante de l'histoire générale de la civilisation. Le point d'ancrage de cette démarche est « la civilisation », sans qu'il soit toujours clair s'il s'agit de la vie civilisée par opposition à la barbarie ou d'une civilisation (exclusivement occidentale pour Comte) parmi d'autres. La science est conçue comme un mode d'activité intellectuelle, « un des facteurs essentiels du progrès vers la civilisation ${ }^{23} »$, dont le développement est étudié en relation avec d'autres. Son histoire générale, intégrée dans le tableau du mouvement intellectuel, comme dit Tannery, ou dans l'histoire culturelle, comme nous pourrions peut-être le dire, est susceptible d'intéresser un vaste public.

Les interrogations de Tannery, telles quelles ont été publiées en 1904 par Berr, se situent clairement au niveau de l'historiographie. Ce sont celles de quelqu'un placé devant la tâche de rédiger une histoire des sciences et de composer un récit historique. Qui écrit quelle histoire, dans quel but et pour quels lecteurs? Ce sont ces questions qui sont à l'origine des critères qui définissent histoires particulières et histoire générale.

Selon que l'histoire des sciences est faite par les savants, les historiens ou les philosophes, les buts qui lui sont assignés divergent. Le savant souhaite étudier «la filiation des idées et l'enchaînement des découvertes », comparer ses conceptions à celles de ses «précurseurs» afin d'orienter le travail futur dans une direction novatrice. L'historien veut « compléter sous le rapport scientifique le tableau du mouvement intellectuel pour telle civilisation ou pour telle époque donnée ${ }^{24} »$, alors que le philosophe s'intéresse aux questions de méthode et à l'évolution des idées scientifiques.

Si l'histoire particulière, comprise par les seuls spécialistes, est au service de l'ars inveniendi, l'histoire générale doit répondre à la double attente des historiens et des philosophes. Quel est alors le lien entre ces deux types d'approche? Dans l'esprit de Tannery, l'histoire générale des sciences sera composée avec des éléments exclusivement fournis par les histoires particulières, auxquelles incomberait la tâche d'élaborer les matériaux bruts tirés des documents originaux. Elle doit être la synthèse des histoires particulières des sciences. Mais qu'est-ce que la synthèse?

22. Berr a publié cette leçon sous le titre «De l'histoire générale des sciences », voir TANNERY, 1904, cité ici in TANNERY, 1912-1950, t. X. Tannery y développe de manière très programmatique ses idées sur une telle histoire. Cette leçon devait servir d'introduction à un ouvrage mettant en œuvre les grandes lignes qui y sont décrites.

23. TANNERY, 1912-1950, t. X, p. 163.

24. TANNERY, 1912-1950, t. X, p. 167. 
Tannery, le 7 avril 1902, avait écrit à Berr :

«[...] il me semble que parmi les collaborateurs [de la Revue de synthèse historique], il y en a très peu qui s'entendent sur le sens du mot synthèse et que plusieurs n'y voient guère que la coordination des efforts pour obtenir des résultats analytiques plus complets et plus détaillés ${ }^{25}$. »

Or, pour lui, la synthèse historique apporte quelque chose de radicalement nouveau (même s'il est très peu loquace sur sa nature). D'après l'étymologie, explique-t-il dans le texte publié en 1904, synthèse serait identique à composition. La réunion et la coordination des éléments obtenus par l'analyse des documents que consulte l'historien constituent la synthèse. Celle-ci, en histoire, ne reproduit pas, comme en chimie, un composé semblable à ceux qui ont été analysés. Bien au contraire, l'interprétation et le rapprochement d'éléments synthétisés devraient permettre de formuler des lois générales et de dégager des concepts historiques. Mais là-dessus, Tannery reste prudent et admet la possibilité d'un simple récit historique (écrit ad narrandum, non ad probandum). Il est d'ailleurs conscient de la valeur relative de toute synthèse, c'est une affaire de point de vue et de perspective, comme Enrico Castelli Gattinara ${ }^{26}$ l'a souligné en commentant la conception de l'histoire de Tannery. D'où l'importance des conjectures dans son travail d'historien. Placé là où il est, l'historien peut formuler des hypothèses qui permettront une interprétation, plus cohérente à ses yeux, des documents qu'il a réunis. Mais, le changement d'observateur ou de point de vue modifie l'interprétation. Tannery répète le « mot de Taine, les textes sont des soldats »; pour ajouter, «je n'aurais aucune peine à les aligner et à les diriger dans mon sens ${ }^{27} \gg$.

De plus, aucune histoire n'est jamais définitivement achevée. La découverte de nouveaux documents et les changements de perspective amènent des remaniements et des refontes continuels. D'où l'accent mis par Tannery sur la recherche et l'étude de documents, d'une part, et sur la formulation de questions, nourries de connaissances modernes, susceptibles de renouveler les interprétations de ces textes, de l'autre :

«C'est le sort réservé à tout travail d'érudition de ne valoir que pour quelques générations; amassons au moins le plus de matériaux et, pour cela, remuons le plus d'idées qu'il nous sera possible. Nos petits-neveux en profiteront ${ }^{28}$. »

25. Lettre publiée par Delorme, 1954, p. 301.

26. Castelli Gattinara, 1998, p. 171-172.

27. TANNERY, 1912-1950, t. XI, p. 608.

28. TANNERY, 1912-1950, t. XI, p. 364. 
Le contexte dans lequel Tannery a élaboré cette approche est fortement marqué par les idées de Berr sur la synthèse ${ }^{29}$. Mot d'ordre en faveur de l'unité du savoir rationnel, la synthèse permet de dépasser les cloisonnements disciplinaires et de mettre en évidence les connexités des questions. Opposée à toute systématisation, qui impose une vue a priori, la synthèse doit se fonder exclusivement sur des connaissances acquises, qu'elle rassemble et élabore. Elle est donc connaissance ouverte, dynamique, tendance vers l'unification, qui est, pour Berr, une loi fondamentale de l'histoire. Mais comment mettre en œuvre cette synthèse (toujours provisoire, le sens de l'histoire n'étant saisissable que par l'histoire tout entière)?

Tannery, fidèle à sa devise En temps et lieu, en envisage deux modes, selon la diachronie et selon la synchronie, ou mieux, selon l'ordre des temps ou selon les disciplines et spécialisations plus ou moins poussées. Il insiste toujours sur la nécessité de recourir aux documents originaux, d'en tirer par analyse des éléments généraux ou spéciaux et de leur faire subir une première élaboration synthétique. Celle-ci constitue soit une histoire particulière d'un sujet scientifique en suivant son développement chronologique, soit une monographie concernant une époque déterminée.

La réunion de toutes les histoires particulières spéciales rangées suivant un ordre de matières rationnel constitue l'histoire spéciale totale (à la Cantor), c'est-à-dire l'histoire de toutes les disciplines et sous-disciplines scientifiques rédigée pour les spécialistes de chacune d'elles. Lorsque, au contraire, on réunit par ordre chronologique les histoires générales des sciences pour les époques successives d'une civilisation particulière, on obtient l'histoire générale des sciences pour cette civilisation. L'histoire générale totale embrasserait, quant à elle, toutes les civilisations.

Alors que chaque discipline scientifique a sa logique propre juxtaposée à d'autres dans l'histoire spéciale totale, l'histoire générale des sciences fait intervenir synthétiquement une époque. Parmi les éléments généraux, Tannery compte l'inventaire raisonné de ce que contiennent les documents, la biographie des savants, tout ce qui est relatif soit aux actions réciproques des sciences les unes sur les autres, soit aux influences exercées sur le progrès ou la stagnation scientifique par les milieux intellectuel, économique et social. Il importe de reconstituer autour des grands savants le cercle des idées qu'ils ont trouvées, de porter son attention pour chaque époque sur le niveau de l'enseignement à ses différents degrés, sur le mode de diffusion des idées et de marquer aussi bien les traits caractéristiques du milieu intellectuel, que ceux qui singularisent les génies supérieurs.

De l'état de toutes les disciplines à une époque donnée, on peut induire des caractéristiques décrivant cette époque. C'est ce que Tannery avait

29. Voir à ce sujet Biard, Bourel et Brian, 1997, et Castelli Gattinara, 1998. 
tenté dans ce qu'il considère en 1904 comme une esquisse d'une « Histoire générale des Sciences en Europe depuis le XIV ${ }^{\mathrm{e}}$ siècle jusqu'à 1900 », c'està-dire dans les notices rédigées pour l'Histoire générale du IV siècle à nos jours (1893-1901) ${ }^{30}$ d'Ernest Lavisse et d'Alfred Rambaud. La science du Moyen Âge y est caractérisée de livresque ${ }^{31}$, puisqu'elle s'appuie sur la seule autorité des textes. Pour la première moitié du XvI ${ }^{\mathrm{e}}$ siècle, l'histoire des sciences est dite n'être que celle de la médecine. Nullement spécialisé, le médecin vise la science universelle : il apprend les mathématiques pour l'astrologie et les langues anciennes pour étudier les auteurs dans le texte. La poussée décisive en matière de sciences se situe au début du XVII ${ }^{\mathrm{e}}$ siècle et se produit dans un milieu étranger au monde universitaire. En France, c'est le monde parlementaire. Le trait saillant de l'évolution des sciences pendant la seconde moitié du siècle est que, désormais, il n'y a plus de séparation tranchée entre les sciences naturelles, d'un côté, et les sciences mathématiques et physiques, de l'autre. L'époque créatrice $d u \mathrm{XVII}^{\mathrm{e}}$ siècle est suivie ${ }^{32}$ par une période de foisonnement tous azimuts et d'exploration de nouveaux moyens mis en place, période qui se clôt par des tentatives de coordination, comme l'Encyclopédie de Diderot et D'Alembert ou l'Histoire des mathématiques (1758) de Jean-Étienne Montucla. Au XIx ${ }^{\mathrm{e}}$ siècle, la science se diffuse largement à travers la forte organisation de l'enseignement supérieur porté sur la recherche et aussi par ses applications industrielles. Le siècle s'achève, pour Tannery, en pleine activité scientifique :

30. Dite Grande Encyclopédie. Les contributions de Tannery ont été réunies in TANNERY, 1912-1950, t. XVII, p. 123-434.

31. TANNERY, 1912-1950, t. XVII, p. 144-145, va jusqu'à parler à ce sujet de « grand vice de la science du Moyen Âge ». Il met l'accent sur l'absence de démonstration et de méthodes d'exposition rigoureuses. Il est incontestable, dit-il en résumé, «que si, pendant cette longue période, la science proprement dite ne progresse point, l'esprit humain subit une évolution marquée et qu'il ne reste nullement emprisonné dans le cercle des idées de l'Antiquité. L'héritage qu'il a reçu et auquel il s'attache fortement est, à bien des égards, un embarras, comme un bâton trop lourd dont l'enfant veut s'aider pour gravir la pente escarpée; la gêne apparaît dans ses tâtonnements incertains, et il n'évite ni les faux pas ni les chutes, mais la marche n'en sera que plus assurée, lorsque l'adolescent exercé ne sentira plus le fardeau ».

32. TANNERY, 1912-1950, t. XVII, p. 295, décrit les caractères généraux du mouvement scientifique pendant le XVIII ${ }^{\mathrm{e}}$ siècle de façon pour nous, post-kuhniens, très intéressante : « À toute époque créatrice, comme le XVII ${ }^{\mathrm{e}}$ siècle, succède une période où se déroulent normalement les conséquences des principes dus aux génies novateurs. Pendant cette période, les efforts individuels divergent plutôt qu'ils ne se concentrent vers un même but; car il s'agit de reconnaître, dans toutes les directions, jusqu'où peut conduire le nouveau moyen à employer. Le terme du développement est marqué par des tentatives de coordination de l'ensemble des résultats acquis, travail qui réclame des génies d'une autre nature, mais d'une puissance au moins égale à celle des novateurs proprement dits. Ces coordinations, satisfaisant l'esprit de recherche, ont à leur tour pour conséquences, soit un arrêt du progrès scientifique, comme on l'a vu dans l'Antiquité, soit un changement de direction générale, quand il se produit heureusement en même temps, ainsi que cela est arrivé à la fin du XVIII ${ }^{\mathrm{e}}$ siècle et au commencement du $\mathrm{XIX}^{\mathrm{e}}$, une nouvelle poussée rénovatrice. » 
«[...] sa fin ne sera certainement pas pour le futur historien la clôture d'une période $^{33}$. »

Dans un exposé, bref et qui n'est jamais technique, Tannery relève le défi de caractériser le mouvement des idées scientifiques, les méthodes et les disciplines dominantes pour chaque époque, la périodisation lui étant imposée d'avance par le découpage de la Grande Encyclopédie. Le récit est vivant, la volonté de dégager des traits saillants, de ne pas simplement juxtaposer les éléments spéciaux tirés des documents, mais de donner une vue synoptique est évidente et la plupart du temps aboutie. Tannery avait justement reproché au Vorlesungen über Geschichte der Mathematik de Cantor d'être une addition de multitudes de sources hétérogènes. Tannery écrit à une époque où les discussions sur la nature de l'histoire des sciences sont vives. Cantor a à soutenir des controverses avec Zeuthen et avec Eneström, l'éditeur de Bibliotheca mathematica. L'enjeu de la première ${ }^{34}$ porte sur l'interprétation des sources mathématiques. Doit-on se fier davantage à des commentateurs tardifs ou à la reconstruction d'un mathématicien moderne ? Cantor et Eneström débattent ${ }^{35}$ des différentes manières d'écrire l'histoire des mathématiques et introduisent plusieurs distinctions selon que l'historien tient compte de circonstances extérieures au développement mathématique ou non, selon qu'il écrit pour le mathématicien ou le grand public : histoire scientifique versus histoire littéraire (Eneström) ou histoire des «mathématiques » versus « histoire » des mathématiques (Cantor) l'accent étant alternativement mis sur mathématiques ou sur histoire. Même si Tannery n'a que très prudemment pris position, il ressort de la lecture de sa correspondance et de remarques dispersées dans ses comptes rendus que sa sympathie va vers une méthode de reconstruction à la Zeuthen - alliant l'analyse mathématique des résultats du passé et la mise en évidence des connexions logiques entre ces résultats — suivie d'une synthèse qui doit plus à la démarche de Comte et aux idées de Berr qu'au modèle des Vorlesungen de Cantor.

En conclusion de cette partie, il convient de souligner l'ampleur de la réflexion méthodologique menée par Tannery, en interaction avec les débats sur l'autonomie de la discipline au tournant du siècle. Alors que les cadres historiographiques mis en place ont été largement battus en brèche $\mathrm{au} \mathrm{Xx}^{\mathrm{e}}$ siècle, et aussi par Needham comme on le verra ci-dessous, l'insistance sur les crises, les moments de rupture dans l'histoire des sciences, l'alternance de périodes d'innovation et de science dite normale par Thomas Kuhn, a eu une longue et importante postérité. Par ailleurs, et en dépit

33. TANNERY, 1912-1950, t. XVII, p. 433.

34. Voir, à ce sujet, LütZen et PuRKert, 1994.

35. Le débat a lieu dans Bibliotheca mathematica, $3^{\text {e }}$ sér., t. II-IV, 1901-1903. TANNERY, 1912-1950, t. X, p. 173, s’y réfère. 
d'une trop forte restriction de la science objet d'histoire, le développement scientifique a durablement été inscrit par Tannery (sous l'impulsion forte de Comte et en interaction avec Berr) dans le champ culturel. Finalement, Tannery peut aujourd'hui attirer notre attention d'historiens des sciences sur la coexistence de différents types d'histoire et surtout la nécessité de penser, comme il l'a tenté, les liens entre elles. Question d'actualité, s'il en est!

LE PROJET HISTORIOGRAPHIQUE DE JOSEPH NEEDHAM

Si l'idée d'une synthèse occupe également une place centrale dans le projet historiographique de Needham, c'est en un sens très différent, qu'il importe de dégager pour saisir quelques traits saillants de son apport à la discipline.

Un constat conduit le biologiste anglais, après des travaux consacrés pour l'essentiel à l'histoire du domaine qui était le sien, l'embryologie chimique, à se tourner de manière définitive vers l'histoire des sciences en Chine : l'histoire n'a quasiment pas tenu compte des apports chinois en matière de sciences et de techniques et a corrélativement produit l'image d'un savoir scientifique moderne qui serait pour l'essentiel occidental. Dès lors, son objectif est clair : il s'agit de « rendre enfin justice [...] à un grand peuple dont la contribution au progrès de l'humanité a été ridiculement sous-estimée $e^{36} \gg$. On voit ici poindre la rhétorique de revendication dont beaucoup des textes de Needham sont empreints. Il reconnaît même ouvertement ne pas avoir hésité, pour les besoins de la cause, à « avoir été trop partisan dans sa défense acharnée de la civilisation chinoise par rapport à celle des Arabes ou de l'Occident ${ }^{37}{ }$. $^{2}$

Cependant, il ne suffisait pas d'aligner les faits pour imposer à ses collègues historiens ou sinologues l'idée qu'on ne pouvait ni concevoir l'histoire des sciences et des techniques sans la Chine, ni concevoir l'histoire de la Chine sans les sciences et les techniques. Il était nécessaire de modifier les cadres historiographiques eux-mêmes, et c'est là que nous retrouverons l'idée de synthèse.

Comment l'histoire des sciences et des techniques pouvait-elle « rendre enfin justice » au peuple chinois? L'œuvre de Needham offre une collection extraordinairement riche de résultats scientifiques, d'artefacts techniques, essentiels à divers titres pour l'Europe moderne et dont il peut

36. Horolenshaw, 1973, trad. franç., p. 30 (souligné par nous).

37. Horolenshaw, 1973, trad. franç., p. 29 (souligné par nous). 
prouver la présence en Chine de nombreux siècles avant qu'ils ne soient attestés en Occident ${ }^{38}$ : ce sont les fameux «firsts» de la science ou de la technique de l'empire du Milieu ${ }^{39}$. Needham postule donc qu'ils trouvent leur origine en Chine ${ }^{40}$, et doit ferrailler contre des auditoires au sein desquels il se trouve toujours quelque champion pour lutter pied à pied afin de ne pas dépouiller l'Occident de ses priorités. Les mécanismes de défense de ses adversaires s'avèrent si répétitifs, quel que soit le sujet qu'il présente, que le scientifique anglais s'amuse à en ébaucher une typologie : il raille ainsi les réactions qui relèvent du Department of Face-Saving Redefinitions, là où l'on travaille à redéfinir de manière appropriée ce qui fait l'essence d'un concept, d'un objet technique, de sorte à pouvoir opposer sa version chinoise, qui ne satisfait pas à la nouvelle définition, aux versions attestées en Occident, qu'elle caractérise au contraire parfaitement ${ }^{41}$.

Cependant, ces résultats isolés, régulièrement contestés, ne pouvaient suffire à imposer une vision d'ensemble autre de l'histoire. Pouvait-on, en effet, prêcher la prise en compte de la Chine comme d'un acteur majeur de l'histoire des sciences, sans mettre à mal l'idée traditionnelle selon laquelle la constitution des sciences modernes procéderait d'un effort cumulatif, réellement et décisivement engagé dans la Grèce antique, poursuivi tant bien que mal au cours du Moyen Âge, pour reprendre à la Renaissance et culminer à l'époque d'une Révolution scientifique que Needham situait au $\mathrm{XVII}^{\mathrm{e}}$ siècle? Si l'on ne débarrassait pas la représentation du passé qui organisait les recherches en la matière de ce cadre essentiellement linéaire, on pouvait multiplier à loisir les publications montrant que l'empire du Milieu avait devancé les autres civilisations pour tel ou tel acquis : la ligne de force qui continuerait à donner sens aux faits historiques demeurerait la

38. Qu'on pense, pour ne citer que quelques exemples, à ses contributions à l'histoire de l'horlogerie, de l'art de la guerre, qu'on trouvera évoquées in NEEDHAM, 1991, ainsi qu'à ses apports à l'histoire de la chimie in NEEDHAM, 1980, ou de l'étude des plantes in NEEDHAM, 1986. Le tableau de transmissions de techniques de la Chine vers l'Occident, dressé par NeEDHAM, 1954, p. 242, est également parlant.

39. C'est le thème des premiers paragraphes de Needham, 1967. On lit encore in Needham, 1986, p. XXIX, dans l'introduction: «As for biological plant protection, as it is now called, this was an outstanding first for Chinese science and technology » (souligné par nous; l'exemple en question est traité p. 542-543). Needham a sans aucun doute puissamment contribué au développement de cette pratique de l'histoire des sciences comme la recherche des «firsts ».

40. Voir, in Horolenshaw, 1973, trad. franç., p. 29-30, ce que Needham dit à ce sujet de lui-même : «Les preuves de la transmission par les Chinois de leurs découvertes et inventions - reconnues plus précoces qu'ailleurs — manquent parfois, mais il part du principe que le devoir de fournir des preuves revient à ceux qui soutiennent la thèse des inventions indépendantes. » BLUE, 1999, p. 49-51, évoque les critiques adressées à Needham sur sa propension à conclure à des diffusions. NEedHAM, 1954, p. 225 sq., expose toutefois le cadre théorique dans lequel il traitera tout au long de son œuvre des relations entre antécédence et diffusion.

41. Voir, p. ex., Needham, "Science and civilization in China », conférence inaugurale, Distinguished Lecture Series, 13 nov. 1986, University of East Asia, Macau, p. 3, où il croque les scènes en question avec beaucoup de réalisme. 
même, et la Chine serait éternellement vouée à ne jouer qu'un second rôle dans le scénario.

Par-delà la recherche des «firsts » dans tel ou tel domaine, il importait donc de promouvoir une autre vision d'ensemble de l'histoire des sciences et des techniques qui se substituerait aux anciennes représentations et où les résultats concernant la Chine pourraient trouver à s'intégrer. Needham articule une représentation alternative sur la base d'une métaphore, centrale dans son œuvre, qui donne la science moderne comme une mer composée de la contribution de toutes les rivières des traditions scientifiques des civilisations anciennes qui ont coulé vers elle :

"What metaphor then can we use to describe the way in which the medieval sciences of both West and East were subsumed into modern science? The sort of image which occurs most naturally to those who work in this field is that of the rivers and the sea. There is an old Chinese expression about "the Rivers going to pay court to the Sea", and indeed one can well consider the older streams of science in the different civilisations like rivers flowing into the ocean of modern science. Modern science is indeed composed of contributions from all the peoples of the Old World, and each contribution has flowed continuously in it, whether from Greek or Roman antiquity, or from the Arabic world and from the cultures of China and India ${ }^{42}$. »

C'est donc par synthèse des différentes traditions que s'est selon lui constitué le savoir dont dispose l'Europe au temps de la Révolution scientifique - Needham dira plutôt la science œcuménique, important dans le domaine de l'histoire une visée qui lui est chère sur le plan des religions ${ }^{43}$. Le projet désormais assigné à l'histoire des sciences est donc tracé : il s'agira de montrer comment le savoir moderne s'est historiquement constitué par la synthèse de toutes ces traditions, de mettre en évidence la contribution de toutes les civilisations à ce processus, et, en particulier, pour lui,

42. NeEDHAM, 1967 , p. 397 ou p. 271-272 de la traduction française. Les expressions que Needham emploie dans cet article pour figurer cette vision des sciences et de leur histoire se retrouveront à l'identique tout au long de ses publications sur plus de vingt ans. Ainsi, quand il reprend la discussion des questions les plus fondamentales liées à l'ensemble de son œuvre, en introduction de NEEDHAM, 1980, P. XLIII, quand il décrit le projet relatif à l'histoire de la botanique qui a présidé à l'écriture de NEEDHAM, 1986, il mobilise les mêmes termes et les mêmes images. Chemla, 1996, et Chemla 1999, analysent de manière critique et sous deux angles distincts cette représentation, en termes de synthèse, des sciences et de leur histoire.

43. La lutte contre une histoire présentant la science moderne comme le fruit d'une progression unique s'inscrit pour Needham dans le dispositif visant à rendre justice à la Chine : les premiers paragraphes de NEEDHAM, 1973, trad. franç. p. 340, le montrent à l'évidence. Il articule explicitement dans ce texte, comme in Horolenshaw, 1973, p. 32-35, la représentation alternative qu'il avance et le projet œcuménique auquel il souscrit en matière de religion. Needham diagnostique d'ailleurs, dans cette manifestation de l'européocentrisme, des raisons qui tiennent tant à l'histoire des religions qu'à l'histoire politique et économique de l'Occident, voir NeEdHam, 1973, trad. franç., p. 341. 
d'éclairer l'apport de la Chine. Cette nouvelle écriture de l'histoire n'est pas de seule érudition : elle nourrit un plaidoyer en faveur du droit égal pour tous à la surface de la planète de bénéficier des bienfaits de la science et de participer à la recherche. Elle accompagne, en effet, la volonté politique de mettre en place un nouveau régime international de pratique des sciences telle qu'elle s'incarne après guerre à l'Unesco ${ }^{44}$.

Ainsi Needham prend la science moderne comme point de référence dans son travail historiographique ${ }^{45}$ et s'intéresse donc, corrélativement, pour l'essentiel à la période qui précède le XVII ${ }^{\mathrm{e}}$ siècle $^{46}$. Dans la mesure où il se donne comme tâche principale de montrer comment ce savoir scientifique se compose de contributions venues de partout dans le monde, on comprend d'autant mieux que les catégories de «précurseur», d' "antécédent » soient très régulièrement sollicitées dans ses écrits.

En des temps où l'histoire s'attache à resituer les productions scientifiques et les artefacts techniques dans les contextes locaux de leur émergence et de leur utilisation, en des temps où la notion de «précurseur » est considérée, à juste titre, avec suspicion, on pourrait être tenté de conclure que le travail de Needham appartient à une époque révolue de l'histoire des sciences. C'est vrai, pour partie ${ }^{47}$. Il convient peut-être pourtant de ne pas trop se hâter et d'examiner les avantages inhérents au dispositif dans le contexte duquel choisit d'œuvrer un Needham, conscient au plus haut point du lien intime qui unit une vision, une pratique de l'histoire des sciences et un projet politique pour l'humanité ${ }^{48}$. Par exemple, pour pertinente qu'elle soit, la critique de la notion de précurseur, que Needham n'a pas manquée de rencontrer — ne serait-ce que dans le vif de l'action, nous l'avons évoqué —, ne lui apparaît-elle pas comme la dernière arme forgée pour les

44. Petitjean, 1999, discute de la relation que cette mutation de la représentation des sciences et de leur histoire entretient avec la tentative de promouvoir de nouvelles formes de collaboration scientifique internationale après guerre. Il revient, en particulier, sur le rôle joué par Needham entre 1946 et 1948 dans la mise en place, pour les sciences, de programmes analogues à ceux qui se définissaient dans d'autres domaines. Voir également BLUE, 1999, p. 53, 56-57 et 64.

45. Qu'il s'agisse là du choix conscient et délibéré d'un Needham qui mesure certaines limites de la démarche, on peut s'en convaincre en lisant NeEDHAM, 1980, p. XLIII-XLIV. Ces pages sont l'aboutissement d'une discussion où NeEdham, ibid., p. XXXVIII-XLI examine de manière critique la position de Said Husain Nasr qui propose de ne pas juger les sciences de l'ensemble des civilisations à l'aune de la science moderne, mais du «point de vue islamique ».

46. Il conviendrait de nuancer dans la mesure où Needham situe à des époques différentes selon les disciplines le moment auquel le savoir devient œcuménique. Voir NeEDHAM, 1967, et Chemla, 1996.

47. Chemla, 1999, met en évidence des pans entiers de faits relatifs aux sciences en Chine que cette pratique de l'histoire des sciences conduirait à négliger.

48. Needham, 1973, exprime en des termes particulièrement vifs les dangers qui sont attachés à une position européocentriste en histoire des sciences. Sur les corrélats contemporains concrets des positions de Needham, voir également Blue, 1999, p. 60 et 64-67. 
besoins de la cause par les défenseurs de l'idée que la science serait par essence occidentale?

Mais revenons au dispositif d'ensemble et examinons-en les corrélats : à opérer de la sorte, Needham ne peut mettre en évidence que ce qui, des résultats scientifiques et techniques venus de Chine, avait vocation à l'universel, par-delà les singularités locales. Il importe d'y insister : Needham ne nie pas une forme de relation entre des résultats scientifiques, des élaborations techniques, et des «cultures » en contact avec lesquelles ils sont mis au point. Il va même jusqu'à souligner : «It is no less important to be aware that every anticipatory feature of a pre-modern system of science had its Yin as well as its Yang side, disadvantages as well as advantages $^{49}$. » Mais il tient à affirmer, par contraste, la capacité de ces résultats et de ces élaborations à circuler et à être partagés par tous, comme lorsqu' on lit sous sa plume :

«[... the ancient and medieval sciences (though bearing an obvious ethnic stamp) were concerned with the same natural world and could therefore be subsumed into the same oecumenical natural philosophy ${ }^{50}$. »

L'existence de spécificités « locales » ne s'oppose donc pas pour lui à la potentielle universalité des résultats produits, comme cela ressort également de la question qu'il pose :

«When in history did a particular science in its Western form fuse with its Chinese form so that all ethnic characteristics melted into the universality of modern science ${ }^{51}$ ?»

On le constate, d'une part, c'est le fait que toutes les traditions anciennes et médiévales s'attachaient à décrire le même monde qui garantit leur possible fusion en une même entité; d'autre part, par opposition avec elles, la science moderne est pour lui universelle. L'argument qu'il avance pour étayer cette thèse, à savoir que la science moderne s'exprime dans le langage universel des mathématiques, mériterait cependant d'être élaboré plus avant $^{52}$. Par ailleurs, la question que Needham pose met en évidence un

49. Needham, 1980, p. XLII. Cette déclaration, relevons-le, fait partie de l'argumentation développée dans ces mêmes pages de NEEDHAM, 1980, que nous avons déjà rencontrées. Needham y justifie son projet en histoire des sciences et précise ce contre quoi il a pris cette forme. Nous y revenons infra, p. 385-387.

50. Needham, 1973, p. 418 (souligné par nous). Blue, 1999, p. 32 et 46, signale le parallèle avec les positions de Sarton, et identifie chez tous deux l'influence de Comte.

51. NeEdham, 1973, p. 397. Cette opposition, ce face-à-face, entre Occident et Chine est récurrent dans la manière dont Needham aborde l'histoire des sciences, et l'on peut faire l'hypothèse qu'il est induit par la revendication de restituer à la Chine des priorités trop souvent hâtivement attribuées à l'Occident.

52. En raison des limites imparties à cet article, nous renonçons à développer ce dernier point. Voir BluE, 1999, p. 54-56. 
autre de ses présupposés : le fait, pour un résultat élaboré en un point de la planète, d'être repris dans cette synthèse que constitue la science moderne met en évidence son caractère universel par-delà ses « caractéristiques ethniques ». En travaillant donc à l'histoire des sciences du point de vue de la science moderne, Needham se concentrait bien sur ce qui, ayant de fait circulé, s'étant fondu aux autres acquis, est constitué, de par l'histoire, comme universel.

Le modèle alternatif, en opposition auquel il formule sa représentation, apparaît explicitement dans un autre texte où il exprime ses convictions, et c'est là que nous retrouverons une tradition plus proche du programme proposé par Tannery :

«[...] this science is an ultimate value and with its applications forms today a unity into which the comparable contributions of different civilizations (not isolated from each other as incompatible and mutually incomprehensible organisms) all have flowed and flow ${ }^{53}$.»

Ces quelques mots saisissent un ensemble d'idées qui nous paraissent essentielles chez Needham. Tout d'abord, il faut à nouveau y noter la reprise de l'image - centrale pour Needham — selon laquelle les contributions des diverses civilisations ont flué et fluent, telles des rivières. Par ailleurs, la science y est dite aujourd'hui une. L'historien britannique insiste régulièrement sur ce caractère d'unicité et, dans le même temps, réitère souvent l'affirmation selon laquelle l'effort qui, au long de l'histoire, a produit le savoir moderne a été continu, passant d'une civilisation à l'autre sans rencontrer de « barrière ${ }^{54} »$. Pour lui, l'histoire des sciences doit donc être par nécessité globale, et les circulations de connaissances entre civilisations jouent un rôle essentiel afin d'écrire un jour, par exemple dans le cas de la chimie, «the history of man's enquiry into chemical phenomena as one single development throughout the Old World cultures ${ }^{55} \gg$.

La suite de ce texte, aussi bien que l'affirmation entre parenthèses soulignée ci-dessus, disent clairement à quoi cette profession de foi s'oppose :

53. Horolenshaw, 1973, p. 16 (souligné par nous).

54. On retrouve ces deux thèmes dans une déclaration, in NeEdHAM, 1980, p. xxxv (souligné par nous), qui réaffirme les hypothèses fondamentales qui ont présidé à l'écriture de Science and civilisation in China: «Throughout this series of volumes it has been assumed all along that there is only one unitary science of Nature, approached more or less closely, built up more or less successfully and continuously, by various groups of mankind from time to time. This means that one can expect to trace an absolute continuity between the first beginnings of astronomy and medicine in Ancient Babylonia, through the advancing natural knowledge of medieval China, India, Islam and the classical Western world, to the break-through of late Renaissance Europe when, as has been said, the most effective method of discovery was itself discovered. »

55. Needham, 1980, p. Xxxv (souligné par nous). Tous ces thèmes font écho à ceux que mettait en évidence le «projet d'histoire scientifique et culturelle de l'humanité » des débuts de l'Unesco, voir Petitjean, 1999, p. 174-183. 
Needham se refuse à accréditer l'idée qu'on puisse écrire l'histoire des sciences pour une civilisation donnée prise séparément, et c'est contre la version de cette alternative développée par Oswald Spengler dans Le Déclin de l'Occident (1916-1920) qu'il se dresse explicitement ${ }^{56}$. L'opposition entre les deux thèses peut être déclinée terme à terme. Pour Spengler, différentes civilisations produisent différentes sciences aussi bien qu'elles donnent naissance à différentes formes d'art, et les unes comme les autres sont incommensurables - on est aux antipodes de l'affirmation selon laquelle la science serait une, et son histoire continue. À suivre Spengler, donc, la circulation des savoirs de l'une à l'autre ne saurait constituer un temps fondamental du processus historique par lequel la science moderne fut constituée, pas plus qu'on ne saurait inscrire les productions de savoirs des diverses civilisations dans une même histoire. En revanche, les civilisations peuvent dans ce cadre être jaugées à l'aune de leur science. À l'insistance de Needham sur l'unité, la continuité, le mélange, répondent chez Spengler la multiplicité, les fractures, l'incommunicabilité. Dans ce contexte, on peut lire le dispositif dans lequel œuvre Needham comme un rempart contre la tentation de conclure, devant l'existence de spécificités, à une incompatibilité irréductible entre cultures scientifiques.

Needham rejette les corrélats des présupposés de Spengler sur deux plans. D'une part, il explicite la représentation de l'histoire des sciences différente qui irait de pair avec cette autre vision d'ensemble : si l'on doit admettre que diverses civilisations produisirent différentes sciences - si tant est que le mot même soit ici pertinent pour ses adversaires incommensurables, la science moderne ne saurait être que l'une d'entre elles. Et les tenants de ces positions spenglériennes de conclure qu'elle est le pur fruit de l'Occident, tandis que les traditions développées en d'autres points de la planète, loin d'avoir pu y apporter la moindre contribution, représenteraient de simples alternatives qui auraient avorté ${ }^{57}$.

56. «[...] not isolated from each other as incompatible and mutually incomprehensible organisms »: les termes que Needham emploie renvoient à l'historien allemand des années 1930; on peut s'en convaincre en lisant NeEDHAM, 1980, p. XXXv sq., où les thèses en question se trouvent explicitées avant d'être contredites. Blue, 1999, p. 46, témoigne de l'importance majeure que revêtait aux yeux de Needham le fait de combattre ces thèses et du lien qu'il convient d'établir avec l'usage de la «science moderne » dans son travail d'historien.

57. Needham, 1980, développe cette ligne d'argumentation dans son introduction, où l'on peut lire, p. XXXvi (souligné par nous) : "Of course one must not see in traditional Chinese science simply a "failed prototype" of modern science, but the formulation here has surely to be extremely careful. There is a danger to be guarded against, the danger of falling into the other extreme, and of denying the fundamental continuity and universality of all science. This could be to resurrect the Spenglerian conception of the natural sciences of the various dead (or even worse, the living) non-European civilisations as totally separate, immiscible thoughtpatterns, more like distinct works of art than anything else, a series of different views of the natural world irreconcilable and unconnected. Such a view might be used as the cloak of some historical racialist doctrine, the sciences of pre-modern times and the non-European cultures 
Dès lors, la question se pose de savoir comment cette faillite serait advenue. Et, là encore, Needham se démarque clairement des thèses de ceux qu'il prend comme adversaires : il nie la pertinence d'études qui considéreraient le «monde de pensée chinois conçu comme une cellule isolée au sens de Spengler» pour rendre compte, par des «facteurs d'inhibition inhérents », de son échec à produire la science moderne. Certes, il reconnaît l'importance de la question, mais il la formule d'une manière autre, où se lit à nouveau l'ensemble de son dispositif : il se propose de rendre compte de "the failure of China and India to give rise to distinctively modern science while having been ahead of Europe for fourteen previous centuries ${ }^{58} \gg$ et met en avant, à cette fin des facteurs de types économiques et sociaux ${ }^{59}$.

En définitive on peut souligner à quel point la conception de la science moderne comme synthèse de contributions dont il faudrait identifier les origines est tout à la fois centrale dans le projet de Needham et révélatrice de certaines de ses options les plus fondamentales. Pour lui, il ne s'agit là cependant en rien d'un schéma figé ou dépassé : comme il l'explique ${ }^{60}$, ce processus, engagé dès « les débuts de l'astronomie et de la médecine à Babylone », se poursuit aujourd'hui. La médecine lui offre l'exemple d'un domaine où les élaborations chinoises et occidentales attendent toujours d'être fondues en un unique corps de connaissances. Ce cas met en lumière le fait que la synthèse ne serait en aucune façon, selon lui, immédiate. Elle requiert au contraire un travail scientifique dont le produit ne saurait être la simple réunion d'éléments de connaissance d'origines diverses, mais est porteur d'innovation.

being thought of as wholly conditioned ethnically, and rigidly confined to their own spheres, not part of humanity's broad onward march. However, it would leave little room for those actions and reactions that we are constantly encountering, those subtle communicated influences which every civilisation accepted from time to time. » Nous retrouvons bien là l'ensemble des thèmes fondamentaux de Needham, en opposition avec les positions spenglériennes dont il explicite les « dangers » inhérents.

58. NeEdham, 1971; voir Blue, 1999, p. 42, sur l'apparition de cette nouvelle question.

59. WeRSKEY, 1971, p. XXII, montre comment Needham infléchit ses travaux historiques pour y intégrer ce mode d'analyse sous l'influence des communications, au second congrès international d'Histoire des sciences, à Londres en 1931, de la délégation soviétique, ce « petit bataillon de politiciens, d'administrateurs, de scientifiques, d'historiens et de philosophes ». Voir Werskey, 1988, p. 139, auquel nous renvoyons plus généralement pour une analyse du tournant que représente la venue de cette délégation dans l'évolution du groupe de scientifiques anglais de gauche dont Needham faisait partie. WERSKEY, 1971, introduit la réédition des textes de communication de ces participants soviétiques. Dans sa préface à ce même recueil, Needham, 1971, lui-même, établit un lien entre les analyses développées par ces savants soviétiques et la manière dont il convient d'aborder la question de savoir pourquoi la science moderne n'est pas apparue en Chine ou en Inde. NeEdHAM, 1973, trad. franç., p. 345, invite à trouver dans ces approches marxistes les ressources pour « nous, Occidentaux, [...], commencer à renoncer à notre pire défaut, ce redoutable orgueil, le péché contre l'esprit ».

60. NeEDHAM, 1967 et 1980. 
C'est sans doute marqués par la tentative comtienne que Tannery, comme Needham, ont élaboré des représentations globalisantes du développement scientifique sur plusieurs millénaires. Si le point de référence de la réflexion de Needham est la science moderne, synthèse de différentes traditions, Tannery prend la notion de civilisation (ou de vie civilisée), dont la science est un élément intégrant, comme base. La synthèse, conçue par Tannery comme procédé historiographique, doit lui permettre de dégager des traits généraux caractérisant telle civilisation à telle époque sous l'aspect scientifique. Si sa notion d'histoire générale des sciences lui permet de décloisonner au niveau des disciplines, les liens entre les civilisations et les circulations entre elles ne sont pas vraiment thématisés chez Tannery. Needham, quant à lui, respecte rigidement les limites disciplinaires de son époque, mais rejette en revanche toute étanchéité entre les différentes cultures! Toutes, depuis les débuts de l'astronomie en Mésopotamie, contribuent à l'effort continu qui a produit le savoir moderne. À travers le travail de synthèse, la science moderne, une et à vocation universelle, intègre les différents apports. Tannery, très concerné par les phénomènes de décadence, tente de penser non la continuité, mais les ruptures et les crises, qui peuvent être à l'origine de la disparition de pans entiers du savoir, mais aussi d'intenses périodes d'innovation et de création. Ce qu'il est aujourd'hui convenu d'appeler la Révolution scientifique $\mathrm{du} \mathrm{XVII}^{\mathrm{e}}$ siècle prend un relief particulier dans ce schéma. Après une période d'asservissement des sciences au Moyen Âge, les sciences abstraites et démonstratives, telles qu'elles s'étaient constituées en Grèce, renaissent et prennent leur forme moderne. C'est la notion de préscience introduite par Tannery qui permet d'écarter de l'histoire spéciale des sciences tout ce qui précède et suit les Grecs pour magnifier la science déductive grecque puis l'éclosion de la science moderne occidentale. C'est précisément contre une telle conception de l'histoire des sciences que Needham, et après lui beaucoup d'autres, se bat, même s'il ne met pas en cause l'existence de la Révolution scientifique et la périodisation qui en découle. Plutôt que renaissance, elle est pour lui un moment de synthèse par excellence. Tout son programme de recherche s'en déduit : retracer l'origine des contributions qui composent le savoir scientifique moderne.

La confrontation des idées de Tannery et de Needham ne doit cependant pas faire oublier que ces deux historiens des sciences interviennent à plus d'un demi-siècle de distance, dans des contextes intellectuels, professionnels et politiques très différents. Si Tannery a œuvré au tournant du siècle pour l'établissement de la discipline, dans laquelle il n'a pas pu faire carrière, au sens propre du terme, Needham en a bousculé et renouvelé, de 
l'intérieur de l'établissement scientifique, les cadres jugés trop étroits dans un monde marqué par le traumatisme de la Seconde Guerre mondiale, la décolonisation et la mise en place de nouvelles formes de coopération internationale.

Karine Chemla et Jeanne Peiffer (mars 2001). 


\section{LISTE DES RÉFÉRENCES}

Biard (Agnès), Bourel (Dominique) et Brian (Éric), dir., 1997, Henri Berr et la culture du $\mathrm{XX}^{e}$ siècle. Histoire, science et philosophie. Actes du colloque international, 24-26 oct. 1994, Paris, Albin Michel/Centre international de synthèse.

Blue (Gregory), 1999, «Science(s), civilization(s), historie(s). A continuing dialogue with Joseph Needham », in HabiB et RaINA, éd., 1999, p. 29-72.

Boutroux (Pierre), 1938, «L'œuvre de Paul Tannery », Osiris, vol. IV, p. 690-705. Castelli Gattinara (Enrico), 1998, Les Inquiétudes de la raison. Épistémologie et histoire en France dans l'entre-deux-guerres, Paris, Éd. de l'École des hautes études en sciences sociales/Vrin.

Chemla (Karine), 1996, "Que signifie l'expression "mathématiques européennes" vue de Chine?», in Catherine Goldstein, Jeremy Gray, Jim RITTER, éd., L'Europe mathématique. Histoires, mythes, identités/Mathematical Europe. History, myth, identity, Paris, Éd. de la Maison des sciences de l'homme, p. 220-245.

Chemla (K.), 1999, « The Rivers and the sea. Analyzing Needham's Metaphor for the World History of Science », in HabiB et Raina, éd., 1999, p. 220-244.

Coumet (Ernest), 1981, "Paul Tannery: "L'organisation de l'enseignement de l'histoire des sciences" », Revue de synthèse, $\mathrm{III}^{\mathrm{e}}$ sér., 101-102, janv.-juin, p. 87-123.

Delorme (Suzanne), 1954, «Paul Tannery et l'histoire générale des sciences », Revue d'histoire des sciences, t. VII, 4, oct.-déc., p. 297-302.

Grange (Juliette), 2000, Auguste Comte. La politique et la science, Paris, Odile Jacob.

Habib (S. Irfan) et Raina (Dhruv), éd., 1999, Situating the history of science. Dialogues with Joseph Needham, New Delhi, Oxford University Press.

Holorenshaw (Henri), pseudonyme de Joseph Needham, 1973, « The making of an honorary taoist », in Miklas Teich et Robert M. Young, éd., Changing perspectives in the history of science, Londres, Heinemann, p. 1-20, trad. franç. in Didier Gazagnadou, 1991, Joseph Needham, un taoiste d'honneur, autobiographie \& De l'embryologie à la civilisation chinoise, Paris, Éd. du Félin, Unesco, p. 5-35.

ITARD (Jean), 1954, «Sur la méthode de Tannery en histoire des mathématiques », Revue d'histoire des sciences, t. VII, 4, oct.-déc., p. 326-332.

LÜTZEN (Jesper) et PuRKert (Walter), 1994, « Conflicting tendencies in the historiography of mathematics. M. Cantor and H. G. Zeuthen », in Eberhard KNOBLOCH et John McCleary, éd., The History of modern mathematics, Boston, Academic Press, vol. III, p. 1-42.

Needham (Joseph), 1954, Science and civilisation in China, vol. I, Cambridge, Cambridge University Press.

Needham (J.), 1967, « The roles of Europe and China in the evolution of oecumenical science », Advancement of Science, t. XXIV, 119, p. 83 sq., cité ici in 1970, 
Clerks and craftsmen in China and the West, Cambridge, Cambridge University Press, p. 396-418, trad. franç. in NeEdHAM, 1991, p. 270-302.

Needham (J.), 1971, «Foreword», Science at the cross roads. Papers presented to the international congress of the History of science and technology, held in London from June $29^{\text {th }}$ to July $3^{\text {rd }}, 1931$ by the delegates of the U.S.S.R., $2^{\mathrm{e}}$ éd., Londres, Frank Cass and Co., p. vII-X.

Needham (J.), 1973, « The historian of science as ecumenical man. A meditation in the Shingon Temple of Kongosammai-in on Koyasan », in Shigeru NaKayama et Nathan Sivin, éd., Chinese science, Cambridge, MA, The MIT Press, 1973, p. 1-9, trad. franç. in NeEDHAM, 1991, p. 340-348.

Needham (J.), 1980, Science and civilisation in China. Vol. V : Chemistry and chemical technology. Part IV : Spagyrical discovery and invention: apparatus, theories and gifts, Cambridge, Cambridge University Press.

Needham (J.), 1986, Science and civilisation in China. Vol. VI : Biology and biological technololgy. Part I : Botany, Cambridge, Cambridge University Press.

Needham (J.), 1991, Dialogue des civilisations Chine-Occident. Pour une histoire xecuménique des sciences, choix de textes et présentation par Georges MéTAILIÉ, trad. par Francine FÈvRE et Marie-Brigitte Foster, Paris, La Découverte.

Paul (Harry W.), 1976, « Scholarship and ideology. The chair of the general history of science at the Collège de France, 1892-1913 », Isis, vol. LXVII, p. 376-397.

PeIfFER (Jeanne), à paraître, «France », in Joseph W. DAuben et Christoph J. ScRIBA, dir., Writing the history of mathematics. Its historical development, Bâle, Birkhäuser Verlag.

Petit (Annie), 1995, «L'héritage du positivisme dans la création de la chaire d'histoire générale des sciences au Collège de France», Revue d'histoire des sciences, t. XLVIII, 4, p. 521-556.

Petitjean (Patrick), 1999, «Needham, Anglo-French civilities and ecumenical science », in HaBIB et RaINA, 1999, p. 152-197.

SARTON (George), 1938, «Paul Tannery », Osiris, vol. IV, p. 633-689.

Sarton (G.), 1947, «Paul, Jules, and Marie Tannery (with a note on Grégoire Wyrouboff) », Isis, vol. XXXVIII, p. 33-51.

Sarton (G.), 1954, « La correspondance de Paul Tannery et l'histoire de nos études », Revue d'histoire des sciences, t. VII, 4, p. 321-325.

TANNERy (Paul), 1887, La Géométrie grecque, comment son histoire nous est parvenue et ce que nous en savons, essai critique, $1^{\mathrm{re}}$ part. [seule parue], Paris, Gauthier-Villars.

TANNERY (P.), 1893, Recherches sur l'histoire de l'astronomie ancienne, Paris, Gauthier-Villars.

TANNERY (P.), 1893-1901, «L'histoire des sciences en Europe depuis le XIV ${ }^{\mathrm{e}}$ siècle jusqu'à $1900 »$, in TANNERY, 1912-1950, ici t. XVII, Paris, 1950, p. 123-434 [ = Extraits de Histoire générale du IV siècle à nos jours, éd. par Ernest Lavisse et Alfred Rambaud, Paris, 1893-1901].

TANNery (P.), 1904, « De l'histoire générale des sciences », Revue de synthèse historique, t. VIII, 22, p. 1-16, ici repr. in TANNERY, 1912-1950, t. X, 12, p. 163-182.

Tannery (P.), 1912-1950, Mémoires scientifiques, t. I-XVII, éd. par Johan Ludvig Heiberg et Hieronymus G. Zeuthen, Toulouse/Paris, Privat/Gauthier-Villars.

Tannery (P.), 1930, Pour l'histoire de la science hellène. De Thalès à Empédocle, $1^{\text {re }}$ éd. Paris, 1887, ici $2^{\mathrm{e}}$ éd. par Auguste Diès, préf. de Federigo EnRIQues, Paris, Gauthier-Villars.

WersKey (Gary), 1971, «Introduction. On the reception of Science at the cross roads in England », in Science at the cross roads. Papers presented to the international congress of the History of science and technology, held in London 
from June $29^{\text {th }}$ to July $3^{\text {rd }}, 1931$ by the delegates of the U.S.S.R., $2^{\mathrm{e}}$ éd. Londres, Frank Cass and Co., p. XI-XXIX.

Werskey (G.), 1988, The Visible College. A collective biography of British scientists and socialists of the 1930s, $1^{\mathrm{re}}$ éd. Allen Lane, 1978, ici $2^{\mathrm{e}}$ éd. Londres, Free Association Books, 1988, préf. de l'auteur. 